\title{
A influência das ferramentas tecnológicas de comunicação nas relações pessoais dentro do ambiente organizacional
}

\section{The influence of technological communication tools on personal relationships within the organizational environment}

\author{
Cássia Juliano Mello Bacharel em Administração de Empresas. Universidade Feevale (FEEVALE) - Brasil. \\ iaronka.mello@gmail.com \\ Moema Pereira Nunes Doutora em Administração. Universidade Feevale (FEEVALE) - Brasil. \\ moemanunes@hotmail.com
}

RESUMO

A tecnologia está cada vez mais integrada com os processos organizacionais e o uso das ferramentas tecnológicas de comunicação tornou-se habitual no ambiente de trabalho. Este trabalho foi desenvolvido com o objetivo de identificar como as ferramentas tecnológicas de comunicação podem afetar as relações interpessoais e interorganizacionais no ambiente organizacional. Para isso, um levantamento foi realizado com a aplicação de 406 questionários válidos. A análise quantitativa dos dados compreendeu o uso de estatística descritiva, dos testes de Mann-Whitney e Jonckheere-Terpstra para a comparação dos dados e a análise fatorial exploratória. Em um primeiro momento buscou-se identificar quais ferramentas de comunicação são utilizadas no ambiente organizacional. Constatou-se que a ferramenta mais utilizada é o WhatsApp, seguida pelo e-mail e contato telefônico. Em um segundo momento, foi analisada a percepção dos profissionais com relação ao uso destas ferramentas de comunicação e sua influência nas relações no ambiente organizacional. Verificouse que essas ferramentas podem influenciar as relações interpessoais, a realização de atividades pelos profissionais, bem como alguns aspectos da própria organização.

Palavras-chave: Tecnologia. Inovação. Comunicação. Ferramentas tecnológicas de comunicação.

\begin{abstract}
Technology is increasingly integrated with organizational processes and the use of technological communication tools has become commonplace in the work environment. This work was developed with the objective of identifying how the technological tools of communication can affect interpersonal and interorganizational relationships in the organizational environment. For this, a survey was conducted with the application of 406 valid questionnaires. The quantitative analysis of the data included the use of descriptive statistics, the Mann-Whitney and Jonckheere-Terpstra tests for data comparison and exploratory factor analysis. At first, it was sought to identify which communication tools are used in the organizational environment. It was found that the most used tool is WhatsApp, followed by email and telephone contact. Then, the perception of professionals regarding the use of these communication tools in the organizational environment in their relationships in the organizational environment was analyzed. It was found that these tools can influence interpersonal relationships, the performance of activities by professionals, as well as some aspects of the organization itself.
\end{abstract}

Keywords: Technology. Innovation. Communication. Technological communication tools. 


\section{INTRODUÇÃO}

A informação e o processo de comunicação estão historicamente presentes na evolução das organizações. Os processos de comunicação contribuem para o desenvolvimento de formas de inter-relação mais participativas e comprometidas. Entretanto, muitas organizações carecem de maior flexibilidade como uma base na sua permanente transformação, de modo a facilitar a interação entre as pessoas. Neste sentido, a comunicação organizacional ajuda a harmonizar, tanto as relações externas, como as internas, bem como cria um clima de motivação dentro das instituições (DUARTE; BRAGA, 2012). A utilização de ferramentas tecnológicas tornou-se parte essencial deste processo de comunicação nas organizações.

Segundo a Agência de Comunicação Empresarial BH Press (2018), a tecnologia, a internet e a criação de novos softwares modificam constantemente o modo como as empresas trabalham a comunicação interna. Assim, o que funcionava perfeitamente há alguns anos, hoje já não garante os mesmos resultados.

Além disso, as organizações precisam ficar atentas às mudanças culturais para encontrar novas formas de direcionar o processo de inovação e comunicação, sendo importante considerar a percepção de que a cultura organizacional é influenciada pelo homem e o homem é influenciado pela cultura da organização, de forma recíproca e constante (FONTANELLA, 2010). As relações humanas são elementos centrais desta cultura e os processos de comunicação, que percorrem todas as ações organizacionais (DUARTE; BRAGA, 2012), contribuem para o desenvolvimento de formas de inter-relação mais participativas e comprometidas. As organizações podem ser mais flexíveis diante da sua permanente transformação, o que de certo modo pode facilitar a sua interação social de maneira responsável, buscando seus interesses com as condições culturais, econômicas e políticas nas quais se movem (CARVALHO; DUARTE; MACHADO, 2011).

Os avanços da tecnologia da informação desempenham um papel significativo na conformação dos padrões das redes de comunicação entre as empresas, destacando-se suas limitações, como, por exemplo, o fato de não representarem uma possibilidade de substituição das relações "face a face". Apesar disso, algumas organizações passaram a buscar o desenvolvimento de processos inteligentes, para que se tornasse possível otimizar o processo de gerar, armazenar, organizar, disseminar e aplicar o conhecimento dos envolvidos (BRANDÃO; TEMOTEO; CÂNDIDO, 2018).

Com os avanços da inovação tecnológica, percebe-se também uma modificação nos estímulos recebidos em todos os âmbitos da vivência humana. Para Tidd e Bessant (2015, p. 18), o conceito de inovação é "mais do que simplesmente ter boas ideias; é o processo de fazê-las evoluir a ponto de terem um uso prático". A evolução da inovação facilitou o envio e o recebimento de informações, porém o tempo se tornou escasso e precioso. A informação chega muito rápido, não é necessário pesquisar, algumas vezes simplesmente aparecem, tornando mais difícil a conciliação de tarefas e compromissos. É neste contexto que se pode perceber o quanto a tecnologia pode afetar o dia a dia das pessoas e, por vezes, podendo afetar diretamente as entregas dos colaboradores dentro das organizações. As pessoas estão aprendendo a tirar vantagem das inovações tecnológicas, para entender e organizar suas vidas dentro de tantas possibilidades. Ao mesmo tempo em que surgem novas ferramentas, algumas organizações limitam o acesso de seus colaboradores a algumas destas, fundamentadas na potencial redução de eficiência no desempenho profissional. Percebe-se, contudo, que as dificuldades de gestão do trabalho associado ao uso de diferentes ferramentas de comunicação é um desafio tanto para os colaboradores como para os gestores. A grande oferta de recursos tecnológicos para a comunicação, a limitada disponibilidade de tempo para o uso eficiente deles, associados à rotina de trabalho, levam ao objetivo desta pesquisa que consiste em analisar como as ferramentas tecnológicas de comunicação podem afetar as relações interpessoais e interorganizacionais no ambiente organizacional.

Para que fosse possível responder a este questionamento, por meio de um levantamento, a pesquisa investiga quais as ferramentas de comunicação são utilizadas no ambiente organizacional e analisa a percepção dos profissionais com relação ao uso destas ferramentas de comunicação e sua influência nas relações no ambiente organizacional.

Dentro do contexto acadêmico, há limitações no conhecimento científico disponível sobre a relação entre as ferramentas tecnológicas de comunicação e as relações pessoais dentro do ambiente organizacional. 
Esta limitação foi identificada por meio da fundamentação teórica apresentada a seguir. Além disso, o estudo tem relevância prática visto que permitirá que gestores possam ter melhor conhecimento sobre a percepção das pessoas sobre o uso destas ferramentas, o que permitirá melhores tomadas de decisão referente à escolha de quais tecnologias serão utilizadas pelas suas equipes. Cabe ainda justificar que, embora os dados tenham sido coletados e analisados em um momento pré-pandemia, a crise advinda do Covid-19 revolucionou a forma de trabalho. As ferramentas tecnológicas de comunicação tornaram-se elementos centrais no dia a dia de grande parte das pessoas e a melhor compreensão delas pode contribuir com a melhor gestão das equipes.

\section{FUNDAMENTAÇÃO TEÓRICA}

A fundamentação teórica deste artigo compreende os temas relações interpessoais, comunicação organizacional e sua relação com inovação, a Internet e as ferramentas tecnológicas de comunicação

\subsection{Relações interpessoais}

As relações interpessoais deixam o clima organizacional mais positivo e aquecido. Um dos primeiros psicólogos que investigou as relações interpessoais foi Kurt Lewin. Mailhiot (2013) enfatiza que, em uma das pesquisas de Kurt, ele concluiu que tanto a produtividade como a eficiência estão relacionadas com a competência dos membros e com a solidariedade das relações interpessoais. Existem duas condições para que o relacionamento aconteça: a afiliação e a atração. A afiliação é o desejo ou a motivação para estar com determinadas pessoas, independente da afinidade que se tem com elas. A atração se refere à atitude ou emoção positiva que se sente em relação às outras pessoas, fato que pode aproximar indivíduos e instigá-los a estar na sua companhia (AMATO NETO, 2000). A vinculação é caracterizada pelo sentimento de conforto e segurança no âmbito das relações mais íntimas. A integração social reflete os interesses e atitudes em comum, geralmente nas relações com amigos e colegas de trabalho.

A dimensão emocional das relações interpessoais é fundamental para a vida associativa, pois esses processos interativos formam o conjunto de sistemas que a organizam. A forma de convivência é definida pelas condições como os relacionamentos acontecem. Quando ocorrem deteriorações nas relações interpessoais, as relações sociais e interorganizacionais também são afetadas (LEITÃO; FORTUNATO; FREITAS, 2006).

O ser humano, segundo Caetano (2005), é atraído por pessoas que recompensam e ajudam a atender às necessidades sociais; sendo as atitudes, fundamentais nas relações interpessoais. A familiaridade é uma soma de fatores mais importantes, ou seja, o fato de estar perto de alguém que causa sentimentos positivos por um período prolongado aumenta a vontade de estar com essa pessoa mais vezes. Já quando o ser humano é exposto à companhia de pessoas de quem ele não gosta, a antipatia aumenta. Outro ponto que determina as atrações sociais é a proximidade, porém esse fator não define qualidade, pois presencialmente é mais fácil falar com alguém que está ao lado do que atrás, e isso não depende de afinidades (CAETANO, 2005.

\subsection{Comunicação organizacional}

A comunicação se tornou um recurso indispensável estando atrelada ao sucesso organizacional. Trata-se de um fator crítico nas organizações, considerando tanto as tradicionais, como aquelas que buscam modelos de gestão mais avançados. A comunicação é capaz de estruturar os sistemas interno e externo das organizações, devendo ser utilizada de maneira eficaz, considerando a integração com a equipe como parte do processo (MONTEIRO; KARPINSKI; ANGNES, 2015). A comunicação é fundamental para a convivência humana dentro do ambiente organizacional (ZEFFANE; TIPU; RYAN, 2011), independente de seu formato (LACOMBE, 2012).

Martino e Marques (2015) afirmam que a comunicação serve como processo de formação de ações expressivas e atualização da linguagem podendo ajudar a perceber a prática comunicativa como uma dinâmica que promove a fala e ações que sofrem a interferência da linguagem. As interações comunicativas 
configuram-se, para Martino e Marques (2015, p. 17), "como momentos em que diferentes interlocutores usam a linguagem (e produzem linguagem) de modo a produzirem entendimentos sobre algo no mundo objetivo, social e subjetivo".

Uma forma de comunicação, a institucional, surge com a função de elucidar o papel da organização, visando estabelecer relações de confiança e de reputação positiva com todos os públicos da organização por meio da simpatia, credibilidade e confiança (KUNSCH, 2008). Seu objetivo está em revelar o lado público das organizações, estando em contrapartida com a comunicação interna. A comunicação externa está relacionada diretamente com clientes, consumidores, fornecedores, acionistas, empresas concorrentes, sociedade, mídia, governo, órgãos públicos, escolas, sindicatos, entre outros, ou seja, o corpo organizacional interage com o meio externo (MATOS, 2004).

O processo de comunicação interno refere-se às pessoas e suas relações interpessoais no âmbito das organizações (ARAUJO; SIMANSKI; QUEVEDO, 2012). É a informação recebida, processada e transmitida de uma pessoa para outra dentro das organizações. A comunicação interna ajuda na humanização das relações nas organizações, de modo a deixar os colaboradores conscientes do seu lugar, promovendo a integração e a identificação dos objetivos (ARAUJO; SIMANSKI; QUEVEDO, 2012). A comunicação interna promove o aprendizado de ouvir o colega, o aumento da autoestima e os relacionamentos de todos os níveis hierárquicos, além de ser fundamental na realização do processo comunicacional interno eficiente entre os agentes que compõem a organização (MONTEIRO; KARPINSKI; ANGNES, 2015). A comunicação face a face possibilita maior chance de sucesso na conclusão das tarefas (MONTEIRO; KARPINSKI; ANGNES, 2015).

O fluxo de informações da comunicação organizacional pode percorrer diferentes canais (TASSIGNY et al., 2012), visando a geração de resultados e a percepção de diferentes públicos estratégicos de interesse da organização. Neste sentido, os avanços tecnológicos contribuem para que surjam inovações no que tange à comunicação.

\subsubsection{Comunicação organizacional e inovação}

Inovar é antecipar as necessidades futuras e criar produtos e serviços diferenciados. A definição de inovação no ambiente organizacional não está relacionada somente com a invenção de um novo produto, serviço ou tecnologia, mas a uma maneira de se reinventar e encontrar diferenciação diante de um mercado altamente competitivo. Inovar é enxergar as mudanças culturais e se adaptar às exigências do mercado (TIDD; BESSANT, 2015).

Inovações em produtos e serviços tem revelado novas oportunidades de comunicação, seja ampliando disponibilidade, meios de acesso e reduzindo custos. Ferramentas como o WhatsApp não apenas trouxeram novas oportunidades de comunicação no seu surgimento, mas têm apresentado um desenvolvimento constante na busca de ampliação de suas possibilidades de uso. Mas, por ser possível entender a comunicação como um processo, novos métodos podem promover melhorias na produtividade, redução de custos, aumento da vida produtiva de equipamentos e melhoria dos processos (FONTANINI; CARVALHO, 2005).

A tecnologia está cada vez mais integrada com os processos das organizações. Sobre isto, Costa (2011, p. 112) afirma que "a digitalização perpassa, cada vez mais, por diversos processos e, até em atividades corriqueiras do dia a dia, estamos nos confrontando com questões que já não são mais analógicas". Os constantes avanços tecnológicos levam à facilidade de encontrar e divulgar informações por meio da internet, promovendo a globalização de mercados e a integração interna e externa das organizações. Torna-se cada vez mais comum o investimento em tecnologia da informação dentro das organizações (CARDOSO et al., 2017).

Tidd e Bessant (2015, p. 22) afirmam que "a inovação é orientada pela habilidade de fazer relações, de visualizar oportunidades e de tirar vantagem das mesmas. Às vezes, envolve possibilidades completamente novas, como a exploração de avanços tecnológicos [...]". Conforme os autores, a habilidade em fazer relações e o entendimento de como tirar vantagem é fundamental no ambiente inovador que se vive. A forma com que as organizações fazem o gerenciamento da inovação pode afetar diretamente a sua relação com o extenso 
espaço da inovação. Se a organização incentivar os processos de inovação aberta, as chances de ocorrer a troca de conhecimento entre colaboradores, fornecedores e clientes serão maiores (BUENO; BALESTRIN, 2012).

As pessoas contam com a democracia que a internet proporciona, ao mesmo tempo que a criação de conteúdo e alimentação de informações são gerenciadas pelos próprios usuários. Para Almeida (2005, p. 173), "ler telas, apertar teclas, utilizar programas computacionais com interfaces gráficas, dar ou obter respostas ao computador, está para a inclusão digital de forma semelhante à alfabetização no sentido de identificação das letras". As organizações estão totalmente ligadas à internet, de modo que a comunicação ocorre praticamente toda através deste meio, conforme será abordado a seguir.

\subsection{Internet}

No início da década de 1990, a internet entrou na fase de comercialização e o que anteriormente era oferecido pela imprensa, radiodifusão e telecomunicação, passou a ser um meio de produção e acesso, além de proporcionar uma variedade de possibilidades interativas que passam a permitir o comércio e as operações bancárias. Com o passar do tempo, a internet se tornou indispensável nas organizações, devido ao seu potencial estratégico, interferência positiva nos processos organizacionais e, principalmente, pelo aumento da concorrência (CALIFANO, 2018).

Segundo o Relatório da Sociedade 2017, produzido pela União Internacional de Telecomunicações (UIT), as pessoas com idade entre 15 e 24 anos estão pré-dispostas a serem on-line, com um percentual de $70 \%$ contra $48 \%$ do restante da população (CETIC, 2017). No Brasil, segundo o Centro Regional de Estudos para o Desenvolvimento da Sociedade da Informação, o acesso à internet é realidade para $99 \%$ das empresas que possuem de 10 a 249 funcionários e para 100\% das empresas que possuem mais de 249 funcionários (CETIC, 2017).

Diante da infraestrutura da internet que permite o fluxo do conteúdo, Korgaonkar e Wolin (1999) afirmam que a escolaridade está relacionada, positiva e significativamente, com o número de horas gastas por dia na internet, com o percentual de utilização da web para uso pessoal, desviando-se das tarefas, indicando que as pessoas com mais escolaridade tendem a passar menos tempo na internet, talvez devido as suas restrições de tempo.

O Relatório da Sociedade 2017 aponta que, devido ao crescimento da disponibilidade de comunicação da última década, liderado pelo crescimento da telefonia móvel e atualmente colaborado pela banda larga móvel, são os estimulantes para o acesso à internet. A internet é uma rede que promove as interações e relações, tanto econômicas, como sociais, e, por meio de celulares e computadores, proporciona rápido contato entre empresas e clientes (CALIFANO, 2018).

\subsection{Ferramentas tecnológicas de comunicação}

O crescimento extraordinário do uso da internet na última década possibilitou às organizações mudanças consideráveis nos seus processos produtivos, como a criação de grupos virtuais, teletrabalho e eservice, por exemplo. Essas mudanças estão ligadas à arquitetura aberta da internet, o que facilita a inovação. São promovidos novos dispositivos, desenvolvimentos em tecnologia e serviços de acesso, com o intuito de criar oportunidades para as pessoas, governos e empresas (LARA, 2009). Para Alter (2013), dentro das organizações, os colaboradores executam atividades e utilizam recursos por meio de um sistema de trabalho.

Em uma pesquisa divulgada pelo Comitê Gestor da Internet no Brasil (CETIC, 2017), as tecnologias da informação podem ser utilizadas para melhorar o desempenho das rotinas organizacionais, de modo a facilitar processos, otimizar o tempo e reduzir custos. Neste sentido, a digitalização tem o objetivo de reduzir incertezas e simplificar as atividades, melhorando o tratamento das informações e ampliando os conhecimentos organizacionais. Os percentuais de empresas que utilizaram ferramentas, considerando um ranking de ferramentas para acesso remoto, são: $80 \%$ das empresas utilizam e-mail corporativo; $73 \%$ utilizam sistemas de computadores; e, $65 \%$ possuem pastas e arquivos compartilhados (CETIC, 2017). 
O Centro Regional de Estudos para o Desenvolvimento da Sociedade da Informação (CETIC, 2017) divulgou ainda que $79 \%$ das empresas disponibilizaram celulares corporativos e o ranking de atividades realizadas nestes celulares foi: $91 \%$ enviaram mensagens de textos por aplicativos; $83 \%$ enviaram e-mails; $80 \%$ acessaram páginas ou sites pela internet; $72 \%$ enviaram mensagens SMS; $71 \%$ utilizaram aplicativos de mapas; $67 \%$ fizeram fotos e vídeos; $61 \%$ acessaram redes sociais; $44 \%$ utilizaram serviços financeiros / banking via internet ou aplicativos; e, 33\% interagiram com instituições governamentais.

A maioria das organizações utiliza a intranet para divulgar informações dentro das organizações. Segundo dados do CETIC (2017), a "rede de comunicação interna privada de uma organização é baseada em protocolos da internet, é utilizada para compartilhar e trocar informações de uma empresa da mesma forma que ocorre na internet, mas com acesso restrito aos usuários internos". O compartilhamento de informações e documentos podem ficar disponível para todos os colaboradores dentro da intranet.

A transferência de informações pode ocorrer também através das redes sociais, como o Facebook. $O$ chat, popularmente conhecido como bate-papo, é um meio de transferência de informações, tanto escritas, como chamadas de videoconferência, no qual as pessoas podem se ver. O Skype e o WhatsApp são exemplos destas ferramentas. Neste sentido, as informações enviadas ou recebidas podem ser tanto do ambiente externo, como do ambiente interno.

Segundo Costa (2011), as redes sociais são modelos de comunicação multidimensionais, nos quais os indivíduos estão situados como nós na rede, de modo que estabelecem ligações entre eles e com diferentes formas de se relacionar. A vida social e o comportamento das pessoas que convivem são impactados. Nestas redes, a interação, a comunicação e as relações são intermediadas por computadores e seu alcance é intensificado por ser multidirecional. Além disso, sistemas como os ERP - Sistema integrado de gestão empresarial, que serve para armazenar informações financeiras, contábil e operacional, podem ser alimentados pelos colaboradores de acordo as necessidades da empresa.

\section{MÉTODO DE PESQUISA}

Não existe uma metodologia que seja melhor diante das demais, mas deve-se encontrar qual a mais adequada para o estudo a ser realizado (MASCARENHAS, 2012). Considerando o objetivo de analisar como as ferramentas tecnológicas de comunicação podem afetar as relações no ambiente organizacional, optou-se pelo desenvolvimento de um levantamento (survey) com objetivo exploratório e caráter quantitativo.

O levantamento compreendeu uma amostra não probabilística, definida por acessibilidade e conveniência, de 406 respondentes, considerando um universo de pessoas em todo o Brasil que utilizam ferramentas tecnológicas de comunicação para o desempenho de suas atividades profissionais.

A coleta de dados ocorreu no primeiro semestre de 2019 por meio da divulgação do questionário elaborado em ferramenta eletrônica, o qual foi compartilhado em redes sociais pelos pesquisadores, tendo sido compartilhado por outras pessoas para o aumento da abrangência do estudo. Um pré-teste também foi realizado pelo Google Forms. Nesta etapa, os pesquisadores acompanharam a aplicação dos questionários para a identificação de dúvidas. Após dez coletas consecutivas sem que os respondentes tivessem dúvidas, o questionário foi considerado validado para aplicação. As respostas obtidas no pré-teste foram desconsideradas no processo de análise dos dados.

A primeira parte do questionário compreendeu perguntas de caracterização da amostra, a saber: sexo, idade, escolaridade, nível hierárquico e tempo de atuação na empresa atual. Visando o posterior tratamento dos dados, eles foram coletados por meio de perguntas fechadas com categorias de respostas. Os dados de caracterização da amostra foram analisados por meio de estatística descritiva com análise de frequência.

A segunda parte do questionário compreendeu a coleta de informações sobre o uso das ferramentas de comunicação no ambiente de trabalho. As ferramentas disponíveis no momento de coleta de dados previamente identificadas na literatura foram elencadas e uma escala do tipo Likert de 5 pontos foi empregada com o objetivo de mensurar o nível de utilização. As diferentes ferramentas foram apresentadas após a pergunta: “Quais ferramentas de comunicação você utiliza durante o seu horário de trabalho?”. Como resposta, foi solicitada a indicação do nível de utilização sendo 1 discordo totalmente e 5 concordo totalmente. 
Os dados referentes ao uso das ferramentas de comunicação foram inicialmente analisados por estatística descritiva com análise de média e desvio-padrão. Na sequência, as variáveis de caracterização da amostra foram consideradas como variáveis independentes e o uso das ferramentas como variáveis dependentes para que fosse possível analisar a diferença no uso das ferramentas pelos usuários. Considerando que uma das escalas empregadas é de concordância, não foi testada a normalidade dos dados, o que permitiu a aplicação de um teste não paramétrico para comparar a diferença estatisticamente significativa entre os atributos que compõem os blocos do questionário (DANCEY; REIDY, 2006). Para a análise referente ao sexo, por haver duas categorias, o teste de Mann-Whitney foi empregado. Nas demais variáveis, considerando a existência de mais categorias como variáveis independentes, foi utilizado o teste Jonckheere-Terpstra.

A terceira etapa do questionário compreendeu a análise referente à percepção dos respondentes sobre o uso das ferramentas de comunicação no ambiente de trabalho. Como não foi localizada uma escala previamente validada para a coleta de dados, foram elaboradas assertivas a partir do referencial teórico. Estas assertivas são apresentadas na Tabela 1 ao lado dos respectivos autores de referência. Na coleta de dados, cada assertiva foi apresentada aos respondentes e eles foram questionados, em uma escala de concordância que seguia o padrão do bloco anterior de questões, sendo 1 discordo totalmente e 5 concordo totalmente para cada afirmação. 
1 - O uso das ferramentas de comunicação no ambiente organizacional promove o aumento da produtividade e da eficiência.

2 - O uso das ferramentas de comunicação no ambiente organizacional promove a afinidade e aproxima as pessoas.

3 - O uso das ferramentas de comunicação no ambiente organizacional facilita o contato particular não relacionado ao trabalho.

4 - O uso das ferramentas de comunicação no ambiente organizacional auxilia na identificação de pessoas que as assessorem nas suas necessidades.

5 - O uso das ferramentas de comunicação no ambiente organizacional influencia na forma em que as relações acontecem.

6 - O uso das ferramentas de comunicação no ambiente organizacional pode prejudicar as relações interpessoais e isso afeta as relações sociais e interorganizacionais.

7 - O uso das ferramentas de comunicação no ambiente organizacional promove emoções positivas nas relações interpessoais.

8 - O uso das ferramentas de comunicação no ambiente organizacional promove a comunicação, tornando a convivência mais agradável.

9 - O uso das ferramentas de comunicação no ambiente organizacional promove a comunicação e por isso é indispensável.

10 - O uso das ferramentas de comunicação no ambiente organizacional contribui para o sucesso das organizações pelo fato de promover a comunicação.

11 - O uso das ferramentas de comunicação no ambiente organizacional faz com que as emoções não apareçam como realmente são.

12 - O uso das ferramentas de comunicação no ambiente organizacional facilita o entendimento racional das mensagens recebidas e enviadas, deixando o viés emocional de lado.

13- O uso das ferramentas de comunicação no ambiente organizacional promove relações confiantes e positivas através da comunicação institucional.

14 - O uso das ferramentas de comunicação no ambiente organizacional auxilia na consolidação da marca junto aos clientes.

15 - O uso das ferramentas de comunicação no ambiente organizacional é influenciado pelo comportamento dos colegas na comunicação interna.

16 - O uso das ferramentas de comunicação no ambiente organizacional para repassar informações pode alterar o significado da mensagem.

17 - O uso das ferramentas de comunicação no ambiente organizacional é menos eficaz do que a comunicação face a face quando se trata de conclusão de tarefas.

18 - O uso das ferramentas de comunicação no ambiente organizacional facilita a relação com todos os níveis hierárquicos.

19 - O uso das ferramentas de comunicação no ambiente organizacional facilita a relação com todos os níveis hierárquicos e por isso a aumenta a autoestima daqueles que ocupam cargos menores.

20 - O uso das ferramentas de comunicação no ambiente organizacional facilita a absorção de informações que auxiliam na evolução da organização.

21 - O uso das ferramentas de comunicação no ambiente organizacional diminui a frequência das relações face a face.

22 - O uso das ferramentas de comunicação no ambiente organizacional quando focado na inovação de processos pode impactar na redução de custos.

23 - O uso das ferramentas de comunicação que possuem vídeo conferência são mais utilizados dentro do ambiente organizacional do àqueles que somente enviam mensagens de texto.

24 - O uso das ferramentas de comunicação online dentro do ambiente organizacional é mais utilizado do que as ferramentas off-lines.

25 - $O$ uso de redes sociais no ambiente organizacional facilita o desenvolvimento das tarefas.

26 - O uso de sistemas operacionais é um facilitador para a busca de informações dentro do ambiente organizacional.

Fonte: Elaborado pelos autores a partir do referencial teórico (2020).
Referência

Mailhiot (2013)

Amato Neto (2000)

Amato Neto (2000)

Caetano (2005)

Leitão, Fortunato e Freitas (2006)

Leitão, Fortunato e Freitas (2006)

Caetano (2005)

Zeffane, Tipu e Ryan (2011)

Monteiro, Karpinski e Angnes (2015)

Monteiro, Karpinski e Angnes (2015)

Lacombe (2012)

Martino e Marques (2015)

Kunsch (2008)

Kunsch (2008)

Araujo, Simanski e Quevedo (2012)

Araujo, Simanski e Quevedo (2012)

Monteiro, Karpinski e Angnes (2015)

Monteiro, Karpinski e Angnes (2015)

Monteiro, Karpinski e Angnes (2015)

Matos (2004)

OCDE (2005)

Fontanini e Carvalho (2005)

Tidd e Bessant (2015)

Tidd e Bessant (2015)

Costa (2011)

Alter (2013) 
Em um primeiro momento foi realizada a análise descritiva dos dados de forma a identificar os aspectos mais e menos relevantes na perspectiva dos respondentes. As técnicas de média e desvio-padrão foram utilizadas. Na sequência, foi realizada uma análise fatorial exploratória. Dado que o conjunto de assertivas foi elaborado a partir da revisão da literatura, esta análise mostrou-se a mais adequada, segundo Hair Jr. et al. (2009), para que fosse possível a definição da estrutura inerente entre as variáveis que não haviam sido testadas anteriormente.

\section{APRESENTAÇÃO E ANÁLISE DOS DADOS}

A apresentação e análise dos dados está dividida em três etapas. Na primeira é caracterizada a amostra. Na segunda, o foco recai no uso das ferramentas de comunicação pelos respondentes. E, na terceira, é apresentada a análise da percepção dos profissionais com relação ao uso das ferramentas de comunicação nas relações no ambiente organizacional

\subsection{Caracterização da amostra}

A amostra foi composta por 406 pessoas, sendo 253 (62,32\%) do sexo feminino e 153 (37,68\%) do sexo masculino. Nenhum respondente marcou a opção que não queria declarar o sexo. Com relação à faixa etária, houve um predomínio de 42,61\% da amostra na faixa entre 26 a 35 anos. O predomínio foi de respondentes com nível superior incompleto (153 respostas, 37,68\%). Com relação ao nível operacional de ocupação, houve um equilíbrio nas respostas, com 38,18\% atuando em cargos operacionais e 34,24\% em nível tático. Entre os respondentes, 39,66\% atuam há menos de 3 anos na empresa atual. Na Tabela 2 são apresentas as informações de caracterização da amostra.

Tabela 2 - Caracterização da amostra

\begin{tabular}{cccc}
\hline Aspecto & Característica da amostra & Frequência & Porcentagem \\
\hline Idade & Até 25 anos & 78 & $19,21 \%$ \\
$(\mathrm{n}=406)$ & Entre 26 e 35 anos & 173 & $42,61 \%$ \\
& Entre 36 e 45 anos & 93 & $22,91 \%$ \\
& Entre 46 e 55 anos & $10,10 \%$ \\
Escolaridade & Acima de 56 anos & 41 & $5,17 \%$ \\
(n=406) & Nível fundamental incompleto & 21 & $0,99 \%$ \\
& Nível fundamental completo & 4 & $0,99 \%$ \\
& Nível médio incompleto & $1,97 \%$ \\
& Nível médio completo & $14,78 \%$ \\
Nível hierárquico & Nível superior incompleto & 8 & $37,68 \%$ \\
(n=406) & Nível superior completo & 60 & $28,81 \%$ \\
Tempo de & Pós-graduação & 153 & $14,78 \%$ \\
atuação na & Operacional & 117 & $38,18 \%$ \\
empresa & Tático & 60 & $34,24 \%$ \\
(n=406) & Estratégico & 155 & $27,58 \%$ \\
\hline
\end{tabular}

Fonte: Elaborado pelos autores a partir dos dados coletados na pesquisa (2020).

\subsection{Uso das ferramentas de comunicação no ambiente de trabalho}

Com o intenso desenvolvimento das tecnologias da informação, apareceram grandes potencialidades para a mudança organizacional. Entende-se que as tecnologias da informação são as 
ferramentas utilizadas para obter, armazenar, tratar, comunicar e disponibilizar a informação (MASCARENHAS; VASCONCELOS, 2004). A ferramenta de comunicação mais utilizada pelos respondentes é o WhatsApp com média 4,374, seguida pelo e-mail e o contato pessoal. Importante destacar que foi solicitado aos respondentes que indicassem o grau de uso das ferramentas durante o horário de trabalho para o desempenho de suas funções profissionais, e não o seu uso para fins pessoais. Nas Tabelas 3 e 4 são apresentados os dados referentes aos usos das ferramentas de comunicação.

Tabela 3 - Uso das ferramentas de comunicação

\begin{tabular}{|c|c|c|c|c|c|c|}
\hline & Média & $\begin{array}{l}\text { Desvio- } \\
\text { padrão }\end{array}$ & $\begin{array}{c}\text { Sexo } \\
\text { U de } \\
\text { Mann- } \\
\text { Whitney }\end{array}$ & $\begin{array}{c}\text { Significância } \\
\text { Assint. } \\
\text { (bilateral) }\end{array}$ & $\begin{array}{c}\text { Idade } \\
\text { Estatística } \\
\text { observada } \\
\text { de J-T }\end{array}$ & $\begin{array}{c}\text { Significância } \\
\text { Assint. } \\
\text { (bilateral) }\end{array}$ \\
\hline $\begin{array}{l}\text { Aplicativo } \\
\text { de chat } \\
\text { interno }\end{array}$ & 3,089 & 1,6092 & 18163,000 & 0,283 & 29933,500 & 0,738 \\
\hline $\begin{array}{l}\text { Contato } \\
\text { pessoal }\end{array}$ & 4,236 & 0,9207 & 19097,000 & 0,807 & 25365,000 & $0,001^{*}$ \\
\hline e-mail & 4,276 & 1,0808 & 18053,500 & 0,196 & 28754,000 & 0,505 \\
\hline Facebook & 2,571 & 1,4414 & 16557,500 & $0,012^{*}$ & 33713,000 & $0,001^{*}$ \\
\hline Hangout & 1,429 & 0,9128 & 18280,000 & 0,206 & 29724,000 & 0,826 \\
\hline Instagram & 2,426 & 1,4275 & 16767,500 & $0,019^{*}$ & 28487,500 & 0,411 \\
\hline Intranet & 2,773 & 1,6564 & 18717,500 & 0,561 & 29437,500 & 0,951 \\
\hline Skype & 2,461 & 1,6169 & 17047,500 & $0,032 *$ & 29312,000 & 0,869 \\
\hline Telefone & 4,214 & 0,9843 & 19333,000 & 0,984 & 31127,000 & 0,606 \\
\hline WhatsApp & 4,374 & 1,0389 & 19327,500 & 0,978 & 32557,500 & $0,006^{*}$ \\
\hline
\end{tabular}

*Significância estatística ao nível de $5 \%$

Fonte: Elaborado pelos autores a partir dos dados coletados na pesquisa (2020).

Tabela 4 - Uso das ferramentas de comunicação

\begin{tabular}{|c|c|c|c|c|c|c|}
\hline & \multicolumn{2}{|l|}{ Formação } & \multirow{2}{*}{$\begin{array}{c}\text { Nível } \\
\text { hierárquico } \\
\text { Estatística } \\
\text { observada } \\
\text { de J-T }\end{array}$} & & \multirow{2}{*}{$\begin{array}{c}\text { Tempo de } \\
\text { empresa } \\
\text { Estatística } \\
\text { observada } \\
\text { de J-T }\end{array}$} & \multirow[b]{2}{*}{$\begin{array}{l}\text { Significância } \\
\text { Assint. } \\
\text { (bilateral) }\end{array}$} \\
\hline & $\begin{array}{c}\text { Estatística } \\
\text { observada } \\
\text { de J-T }\end{array}$ & $\begin{array}{c}\text { Significância } \\
\text { Assint. } \\
\text { (bilateral) }\end{array}$ & & $\begin{array}{c}\text { Significância } \\
\text { Assint. } \\
\text { (bilateral) }\end{array}$ & & \\
\hline $\begin{array}{l}\text { Aplicativo } \\
\text { de chat } \\
\text { interno }\end{array}$ & 29858,000 & 0,842 & 25495,000 & 0,161 & 10997,500 & 0,272 \\
\hline $\begin{array}{l}\text { Contato } \\
\text { pessoal }\end{array}$ & 31631,000 & 0,206 & 27958,500 & 0,540 & 28126,000 & 0,215 \\
\hline e-mail & 34031,500 & $0,001^{*}$ & 24458,000 & $0,014^{*}$ & 32530,500 & $0,011^{*}$ \\
\hline Facebook & 29825,500 & 0,821 & 28872,000 & 0,189 & 31636,000 & 0,110 \\
\hline Hangout & 32610,000 & $0,010^{*}$ & 27631,500 & 0,678 & 29632,000 & 0,983 \\
\hline Instagram & 31163,500 & 0,403 & 28609,500 & 0,266 & 29336,500 & 0,827 \\
\hline Intranet & 33540,000 & $0,008^{*}$ & 25626,000 & 0,190 & 31280,000 & 0,181 \\
\hline Skype & 33771,000 & $0,003 *$ & 24894,500 & 0,051 & 33063,500 & $0,005^{*}$ \\
\hline Telefone & 30141,500 & 0,979 & 27208,500 & 0,981 & 32943,500 & $0,005^{*}$ \\
\hline WhatsApp & 32414,500 & $0,040^{*}$ & 28578,500 & 0,223 & 31797,500 & 0,051 \\
\hline
\end{tabular}

*Significância estatística ao nível de 5\%

Fonte: Elaborado pelos autores a partir dos dados coletados na pesquisa (2020).

Cada vez mais, as pessoas contam com a facilidade de comunicar-se que a internet proporciona, além disso, a criação de conteúdo e alimentação de informações são gerenciadas pelas próprias pessoas. Para Almeida (2005, p. 173), “ler telas, apertar teclas, utilizar programas computacionais com interfaces gráficas, dar ou obter respostas ao computador, está para a inclusão digital de forma semelhante à alfabetização no sentido 
de identificação das letras". As organizações estão totalmente ligadas à internet, de modo que a disseminação e armazenamento de informações ocorre praticamente toda através das ferramentas tecnológicas.

O e-mail segue como um dos meios mais utilizados para se comunicar, perdendo apenas para o WhatsApp que é o aplicativo mais utilizado para se comunicar conforme os dados desta pesquisa. E, apesar da disponibilidade gratuita da maioria das ferramentas, o contato pessoalmente ainda é bastante usado, ou seja, as pessoas possuem a necessidade de ver as outras.

Nas Tabelas 3 e 4 também é apresentado o resultado da análise dos testes que verificaram a existência de diferença significativa entre os usuários de acordo com as variáveis de caracterização da amostra. Com relação ao sexo, foi identificada diferença significativa no uso das ferramentas Facebook $(\alpha=0,012)$, Instagram $(\alpha=0,019)$ e Skype $(\alpha=0,032)$. O uso do Facebook e do Instagram é mais valorizado pelos respondentes do sexo feminino, ao passo que o Skype mostrou mais relevante para os respondentes do sexo masculino. Analisando o exposto na Tabela 3, pode-se perceber que o sistema de trabalho mais utilizado pelos homens são as ferramentas de envios de mensagens e, além disso, segundo os dados, valorizam mais contatos pessoais do que as mulheres. O sexo feminino é mais diversificado na escolha das ferramentas utilizadas, sendo elas, email, Facebook e Instagram. Embora não seja possível uma extrapolação dos dados, pode-se verificar uma tendência que as mulheres estariam mais dispostas que os homens a conhecer e utilizar outras ferramentas.

A análise da idade permitiu a identificação de diferença significativa no uso do contato pessoal ( $\alpha=$ 001), Facebook $(\alpha=0,001)$, e WhatsApp $(\alpha=0,006)$. O grupo de respondentes mais jovens atribuiu uma importância maior ao contato pessoal (média 4,44 ) em comparação com os grupos de idades superiores. Como pode-se observar na Tabela 3, há quase que uma redução gradual na relevância à medida em que a idade dos respondentes avança. Situação quase oposta percebe-se no uso do Facebook, com uma valorização maior dos respondentes mais velhos. $O$ uso do WhatsApp, embora de grande intensidade em todas as faixas etárias - a menor média foi de 4,12 no grupo mais jovem; sua maior utilização está nos grupos etários intermediários.

A formação gerou diferença significativa no uso do e-mail $(\alpha=0,001)$, do Hangout $(\alpha=0,010)$, da Intranet $(\alpha=0,008)$, do Skype $(\alpha=0,003)$ e do WhatsApp $(\alpha=0,040)$. Korgaonkar e Wolin (1999) afirmam que a escolaridade está relacionada, positiva e significativamente, com o número de horas gastas por dia na internet, com o percentual de utilização da web para uso pessoal, desviando-se das tarefas, indicando que as pessoas com mais escolaridade tendem a passar menos tempo na internet, talvez devido às suas restrições de tempo. Pode-se perceber que o grupo com ensino médio incompleto está com o valor total da média, reafirmando a teoria de Korgaonkar e Wolin (1999) e, sendo assim, o grupo que mais usa o aplicativo. Os grupos que mais utilizam a ferramenta de e-mail, são o com escolaridade superior completo e o com especialização, demonstrando que utilizam a internet para o trabalho. O nível fundamental incompleto se destaca na utilização do Facebook. Entende-se com esse resultado que o nível de escolaridade influencia no tipo de ferramenta que a pessoa tende a utilizar.

Nível hierárquico, por sua vez, mostrou diferença apenas no uso do e-mail $(\alpha=0,014)$, sendo mais frequente pelos profissionais de nível tático. Conforme pode ser observado na Tabela 4 , os profissionais de nível tático utilizam mais o WhatsApp, sendo que a diferença no uso do e-mail foi de apenas 0,01 ponto. $\mathrm{O}$ email foi a ferramenta mais destacada pelos profissionais de nível operacional, enquanto no nível estratégico prevaleceu o uso do WhatsApp.

O tempo de empresa, por sua vez, revelou diferença significativa no uso do e-mail $(\alpha=0,011)$, do Skype $(\alpha=0,005)$ e do telefone $(\alpha=0,005)$. Na Tabela 4 é possível visualizar que o e-mail é mais utilizado pelos profissionais que estão entre 8 e 11 anos na organização, mas que todos os respondentes das demais faixas de respostas também indicaram alto uso da ferramenta. O Skype também foi mais destacado pelo mesmo grupo de respondentes, muito embora a média seja muito inferior, em todos os grupos. Já o telefone apresentou um grande uso pelos colaboradores que possuem 12 anos ou mais de atuação nas empresas.

\subsection{Análise da percepção dos profissionais com relação ao uso das ferramentas de comunicação nas relações no ambiente organizacional}


As relações interpessoais deixam o clima organizacional mais positivo e aquecido. Segundo Kurt Lewin, tanto a produtividade, quanto a eficiência, estão precisamente relacionadas com a competência dos membros e com a solidariedade das relações interpessoais (MAILHIOT, 2013). Na análise geral, os respondentes concordam com Mailhiot (2013), apontando, com maior média, a afirmativa que o uso das ferramentas de comunicação no ambiente organizacional promove o aumento da produtividade e da eficiência. Pode-se perceber que os profissionais de hoje procuram ferramentas para aumentar a sua produtividade e, também, para a realização das tarefas com mais efetividade. Na sequência contatou-se a percepção sobre o uso das redes sociais e sua contribuição para facilitar o desempenho das tarefas, conforme exposto por Costa (2011). A terceira assertiva com maior média refere-se ao apresentado por Monteiro, Karpinski e Angnes (2015), segundo os quais o uso das ferramentas de comunicação no ambiente organizacional é menos eficaz do que a comunicação face a face quando se trata de conclusão de tarefas. Percebe-se aqui um impacto negativo destas ferramentas, em oposição as duas assertivas anteriores. A possibilidade de apoio na redução de custos, aspecto levantado por Fontanini e Carvalho (2005) foi identificado como quarto elemento mais significativo. O quinto impacto mais relevante para os respondentes refere-se ao exposto por Lacombe (2012), para o qual o uso das ferramentas de comunicação no ambiente organizacional faz com que as emoções não apareçam como realmente são. $\mathrm{O}$ Gráfico 1 consolida estes resultados.

Gráfico 1 - Aspectos mais relevantes no uso das ferramentas de comunicação

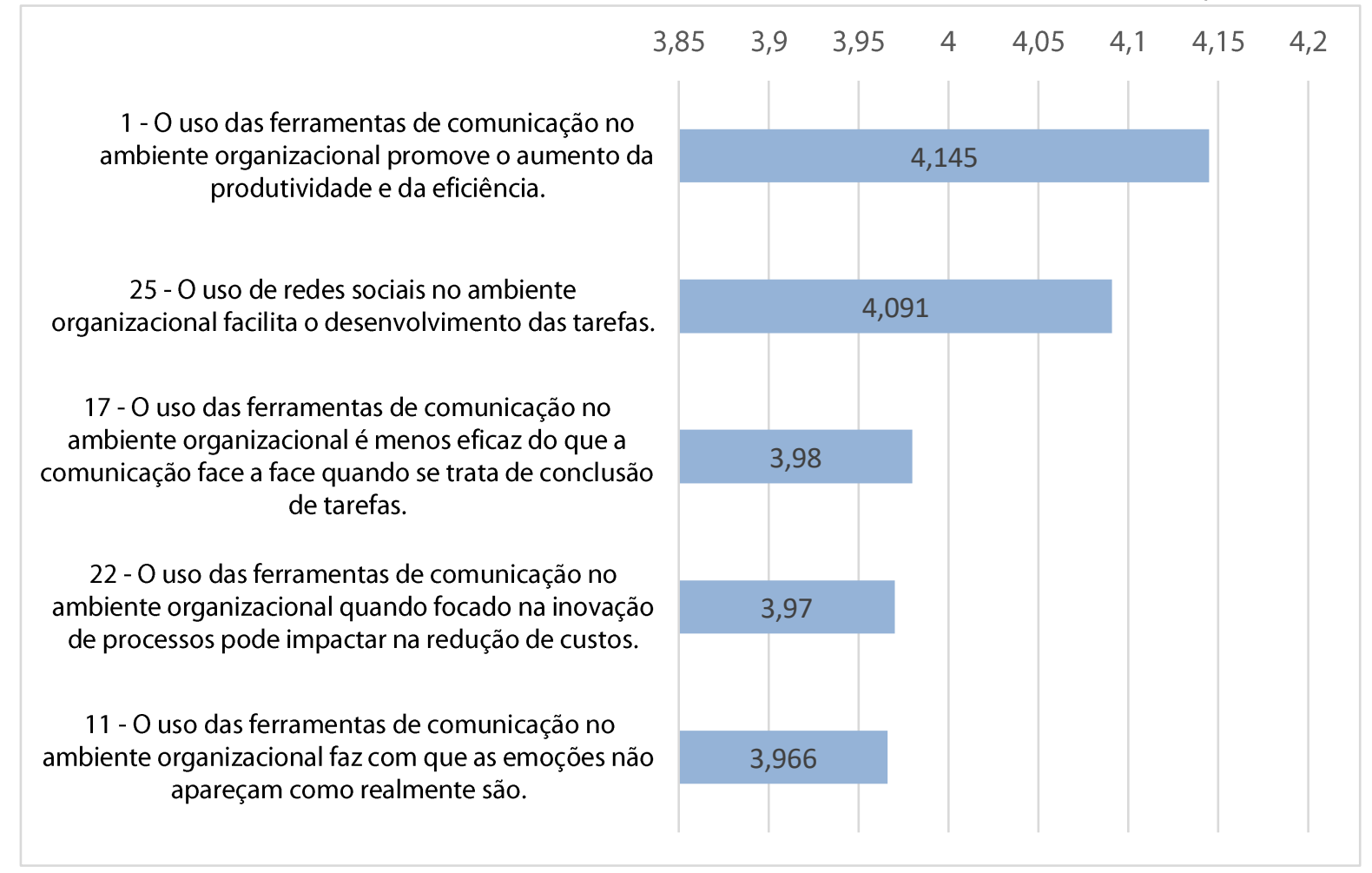

Fonte: Elaborado pelos autores a partir dos dados coletados na pesquisa (2020).

Com relação aos aspectos menos percebidos pelos respondentes destaca-se o exposto por Monteiro, Karpinski e Angnes (2015), para os quais o uso das ferramentas de comunicação no ambiente organizacional promove a comunicação e por isso é indispensável. Contatou-se ainda que a influência no comportamento dos colegas na comunicação interna pelo uso das ferramentas de comunicação no ambiente organizacional, aspecto destacado por Araujo, Simanski e Quevedo (2012), não se mostrou como de grande relevância aos respondentes do estudo. Da mesma forma, o uso das ferramentas de comunicação on-line dentro do ambiente organizacional é mais frequente do que as ferramentas off-lines, conforme afirmação de Tidd e Bessant (2015) a qual obteve baixa relevância para os respondentes, bem como a afirmação de Leitão, Fortunato e Freitas (2006), para os quais o uso das ferramentas de comunicação no ambiente organizacional pode prejudicar as relações interpessoais, afetando as relações sociais e interorganizacionais. Dentre as 26 assertivas, a que 
obteve menor média foi o exposto por Tidd e Bessant (2015) ao afirmarem que as ferramentas de comunicação que possuem vídeo conferência são mais utilizadas dentro do ambiente organizacional do que aquelas que somente enviam mensagens de texto. $O$ Gráfico 2 consolida estes resultados.

Gráfico 2- Aspectos menos relevantes no uso das ferramentas de comunicação

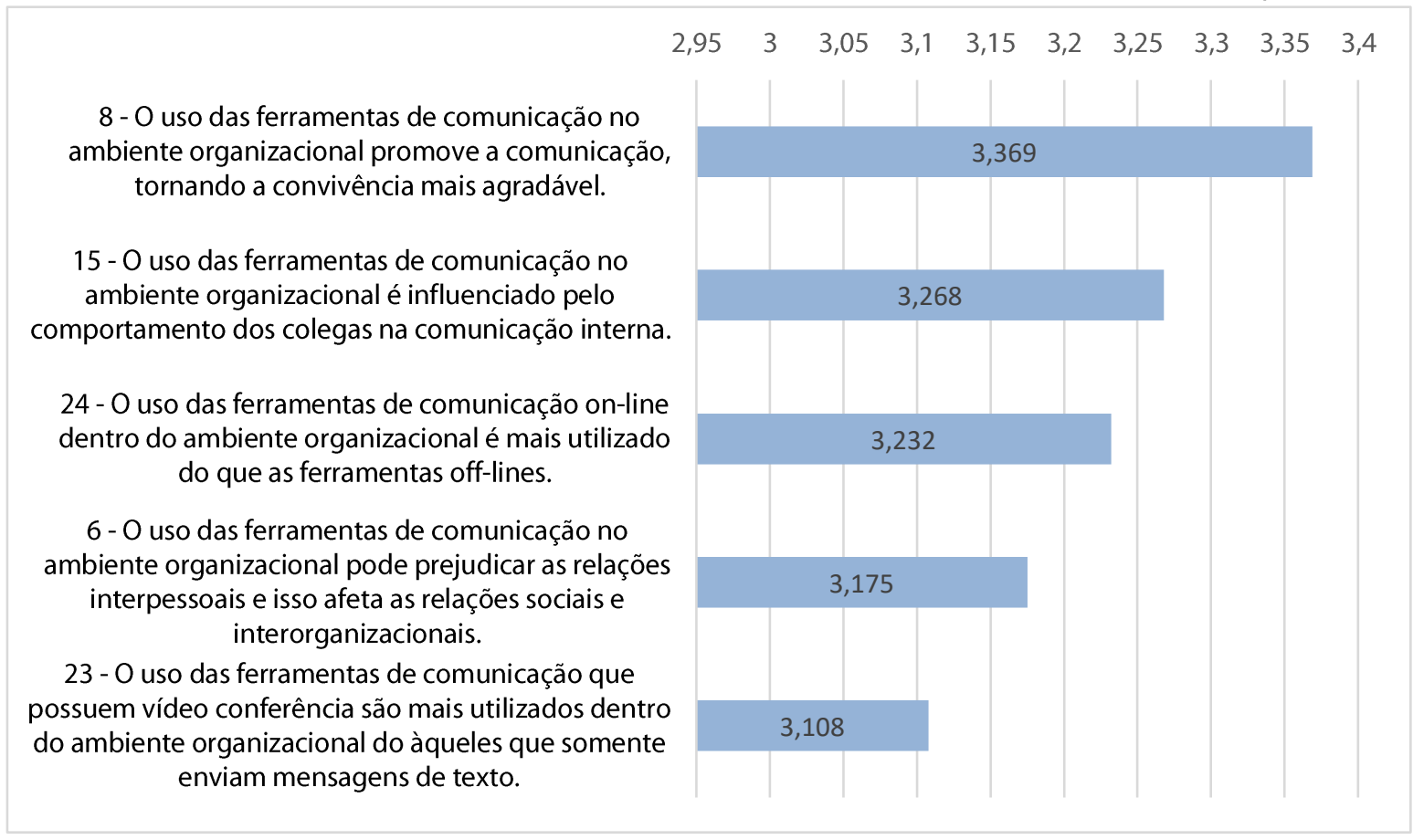

Fonte: Elaborado pelos autores a partir dos dados coletados na pesquisa (2020).

A análise descritiva com média e desvio-padrão de todas as assertivas é apresentada na Tabela 5. Interessante destacar que mesmo a menor média $(3,108)$ pode ser considerada alta em uma escala de 5 pontos. Percebe-se assim que, mesmo que alguns elementos tenham sido indicados como mais relevantes, todos mostraram-se relevantes para os respondentes.

Para o desenvolvimento da análise fatorial exploratória, optou-se pela não identificação prévia de fatores, e pela construção de uma amostra adequada ao número de variáveis. Segundo Hair Jr. et al. (2009), o mínimo deve ser de 10 observações por variáveis. Neste estudo obtiveram-se 15 observações por variável (406 respostas válidas e 26 variáveis). A intercorrelação dos dados foi assegurada pelo teste Kaiser-Meyer-Olkin superior a 0,8 (KMO =0,919) e pela significância inferior a 0,05 no teste de esfericidade de Bartlett $($ sig. $=0,000)$. A análise compreendeu o uso da rotação Varimax com solução rotacionada e cargas inferiores a 0,40 levaram à exclusão das variáveis dada a baixa significância. O processo de análise fatorial compreendeu ainda a análise das comunalidades das variáveis, tendo todas apresentado valores superiores a 0,5 . A soma de rotação de carregamentos ao quadrado revelou uma variância de $56,82 \%$

A partir da análise da matriz de componentes rotativa, apresentada na Tabela 5, é possível observar que as assertivas foram agrupadas em 5 componentes iniciais. Analisando as cargas fatoriais, e excluindo as variáveis que apresentaram cargas cruzadas, constatou-se que os componentes 3 e 5 permaneceram com apenas duas variáveis cada, sendo eliminados da análise. Assim, obteve-se ao final três fatores, referentes aos componentes 1,2 e 4. 
1 - O uso das ferramentas de comunicação no ambiente organizacional promove o aumento da produtividade e da eficiência.

2 - O uso das ferramentas de comunicação no ambiente organizacional promove a afinidade e aproxima as pessoas.

3 - O uso das ferramentas de comunicação no ambiente organizacional facilita o contato particular não relacionado ao trabalho.

4 - O uso das ferramentas de comunicação no ambiente organizacional auxilia na identificação de pessoas que as assessorem nas suas

necessidades.

5 - O uso das ferramentas de comunicação no ambiente organizacional influencia na forma em que as relações acontecem.

6 - O uso das ferramentas de comunicação no ambiente organizacional pode prejudicar as relações interpessoais e isso afeta as relações sociais e interorganizacionais.

7 - O uso das ferramentas de comunicação no ambiente organizacional promove emoções positivas nas relações interpessoais.

8 - O uso das ferramentas de comunicação no ambiente organizacional promove a comunicação, tornando a convivência mais agradável.

9 - O uso das ferramentas de comunicação no ambiente organizacional promove a comunicação e por isso é indispensável.

10 - O uso das ferramentas de comunicação no ambiente organizacional contribui para o sucesso das organizações pelo fato de promover a comunicação.

11 - O uso das ferramentas de comunicação no ambiente organizacional faz com que as emoções não apareçam como realmente são

12 - O uso das ferramentas de comunicação no ambiente organizacional facilita o entendimento racional das mensagens recebidas e enviadas, deixando o viés emocional de lado.

13- O uso das ferramentas de comunicação no ambiente organizacional promove relações confiantes e positivas através da comunicação institucional.

14 - O uso das ferramentas de comunicação no ambiente organizacional auxilia na consolidação da marca junto aos clientes.

15 - O uso das ferramentas de comunicação no ambiente organizacional é influenciado pelo comportamento dos colegas na comunicação interna

16 - O uso das ferramentas de comunicação no ambiente organizacional para repassar informações pode alterar o significado da mensagem.

17 - O uso das ferramentas de comunicação no ambiente organizacional é menos eficaz do que a comunicação face a face quando se trata de conclusão de tarefas.

18 - O uso das ferramentas de comunicação no ambiente organizacional facilita a relação com todos os níveis hierárquicos.

19 - O uso das ferramentas de comunicação no ambiente organizacional facilita a relação com todos os níveis hierárquicos e por isso a aumenta a autoestima daqueles que ocupam cargos menores.

20 - O uso das ferramentas de comunicação no ambiente organizacional facilita a absorção de informações que auxiliam na evolução da organização.

21 - O uso das ferramentas de comunicação no ambiente organizacional diminui a frequência das relações face a face.

\begin{tabular}{|c|c|c|c|c|c|c|}
\hline \multirow[b]{2}{*}{ Média } & \multirow{2}{*}{$\begin{array}{l}\text { Desvio } \\
\text { padrão }\end{array}$} & \multicolumn{5}{|c|}{ Componente } \\
\hline & & 1 & 2 & 3 & 4 & 5 \\
\hline 4,145 & 8351 & & ,608 & & & \\
\hline 3,645 & 1,0387 & 635 & & & & \\
\hline 3,724 & 9416, & 789 & & & & \\
\hline 3,603 & ,9599 & 632 & & 49s & & \\
\hline
\end{tabular}

$3,603,6599,632$

$3,904,8392 \quad 636$

23 - O uso das ferramentas de comunicação que possuem vídeo conferência são mais utilizados dentro do ambiente organizacional do àqueles que somente enviam mensagens de texto.

24 - O uso das ferramentas de comunicação on-line dentro do ambiente organizacional é mais utilizado do que as ferramentas off-lines.

26 - O uso de sistemas operacionais é um facilitador para a busca de informações dentro do ambiente organizacional. 
O primeiro fator compreende assertivas relacionadas com as relações interpessoais. De acordo com Amato Neto (2000), o uso das ferramentas de comunicação no ambiente organizacional promove a afinidade entre as pessoas, facilitando o contato além das atividades relacionadas com a atividade profissional. Leitão, Fortunato e Freitas (2006) ainda ampliam este horizonte ao afirmar que estas ferramentas influenciam a forma como estas relações acontecem, sem distinção de objetivo entre profissional e pessoal. Ainda neste fator encontra-se o impacto positivo do uso destas ferramentas nas relações interpessoais, conforme exposto por Caetano (2006).

O segundo fator, referente ao componente 2 , apresente assertivas relacionadas com a realização das atividades profissionais. Para Mailhiot (2013), o uso destas ferramentas promove o aumento da produtividade e da eficiência. O potencial de redução de custos por meio do uso destas ferramentas é destacado por Fontanini e Carvalho (2005), ao passo que Costa (2011) ressalta a capacidade de facilitar o desenvolvimento de tarefas. Complementando, Alter (2013) destaca que sistemas operacionais facilitam a busca de informações dentro das organizações. Monteiro, Karpinski e Angnes (2015) afirmam, entretanto, que o seu uso é menos eficaz do que a comunicação face a face quando se trata da conclusão de tarefas.

O terceiro fator foi denominado de aspectos da organização. Para Matos (2004) o uso das ferramentas de comunicação no ambiente organizacional facilita a absorção de informações que auxiliam na evolução da organização. Ao mesmo tempo, tem-se a afirmação de Kunsch (2008) de que este uso promove relações confiantes e positivas através da comunicação institucional. Monteiro, Karpinski e Angnes (2015) consideram que este uso facilita a relação com todos os níveis hierárquicos e por isso a aumenta a autoestima daqueles que ocupam cargos menores. Já Para Leitão, Fortunato e Freitas (2006), podem ocorrer prejuízos das relações interpessoais, afetando tanto as relações sociais como as interorganizacionais. Ainda, o uso das ferramentas pode estar relacionado com a diminuição da frequência das relações face a face segundo o Manual de Oslo (OCDE, 2005).

\section{CONSIDERAÇÕES FINAIS}

Os recentes avanços tecnológicos aceleraram a velocidade com que as mudanças têm acontecido, impactando na comunicação dentro e fora das organizações. Surgiram muitos aplicativos para a promoção da comunicação rápida e dinâmica e, com as diversas possibilidades oferecidas pela internet, surgem, também, as dificuldades para lidar com tantas ferramentas.

O estudo permitiu não apenas a identificação das ferramentas de comunicação mais utilizadas, mas também a verificação da existência de diferenças entre o uso das ferramentas de acordo com as variáveis de caracterização da amostra. Muito embora os resultados obtidos neste estudo não permitam a generalização em decorrência do tipo de escala de coleta e do processo de análise dos dados, estes resultados podem ser interpretados como tendências. Assim, a compreensão de que as diferentes ferramentas serão mais ou menos valorizadas no dia a dia dos colaboradores de uma organização pode auxiliar os gestores na seleção daquelas ferramentas que serão empregadas como canais formais da organização, alinhando assim a definição organizacional com a tendência de comportamento dos seus colaboradores.

Uma recomendação de estudo futuro é justamente a ampliação do estudo sobre o uso das ferramentas de comunicação, não apenas identificando as mesmas, mas também buscando compreender o que motiva ou não o uso de cada uma das ferramentas. Este conhecimento permitirá a melhor gestão das ferramentas pelas organizações.

Com relação ao impacto do uso das ferramentas de comunicação, os respondentes destacaram aspectos positivos como a possibilidade de aumento da produtividade e da eficiência, o apoio no desenvolvimento de tarefas e o potencial de redução de custos. Além disto, dentre os aspectos potencialmente negativos foi atribuída maior concordância com relação ao fato de as ferramentas tecnológicas serem potencialmente menos eficazes que a comunicação face a face, bem como o potencial das emoções não aparecerem da forma como realmente são.

Dentre as assertivas, cabe um destaque à que teve a menor média, a qual comparava o uso de ferramentas de texto com ferramentas de vídeochamadas. Considerando que os dados foram coletados em 2019, é preciso ter em mente que naquele momento o mundo ainda não havia começado a experimentar os 
impactos do distanciamento social imposto pelo Covid-19. Após o surgimento do vírus e a consequente pandemia, o uso das ferramentas tecnológicas de informação adquiriu um novo status nas organizações. $O$ uso destas ferramentas precisou ser intensificado, substituindo o contato pessoal, e atributos até então talvez pouco valorizados, como as imagens de vídeo, podem ter adquirido uma relevância superior no contexto das relações tanto pessoais como organizacionais. Assim, percebe-se uma oportunidade de estudo futuro com a aplicação da ferramenta de estudo para analisar a percepção dos colaboradores a partir deste momento em que o uso destas ferramentas se tornou mais relevante.

Destaca-se aqui uma limitação do estudo com relação às ferramentas que foram apresentadas para análise de uso pelos respondentes. A ferramenta Zoom, por exemplo, nem foi listada na coleta de dados em 2019 devido ao uso ainda muito restrito naquele momento. Além disso, ao serem questionados sobre ferramentas como o Instagram e o Facebook, não foram feitos questionamentos específicos sobre o uso delas para compartilhamento amplo de informações e outro específico sobre suas funções de conversa. Estas limitações do estudo precisam ser destacadas para que em estudos futuros estas lacunas possam ser preenchidas.

Ainda analisando a percepção sobre o uso das ferramentas, a análise fatorial exploratória permitiu uma melhor compreensão das relações entre as assertivas, bem como atribuiu maior segurança à análise dos dados visto que não foi encontrada previamente uma escala já validada que permitisse a análise necessária neste estudo. Os resultados obtidos permitiram identificar que o uso das ferramentas de comunicação pode afetar as relações interpessoais, o desempenho das atividades profissionais, bem como aspectos da própria organização na qual os colaboradores atuam.

Neste ponto é importante destacar que uma limitação deste estudo é o fato dele não ter tido como objetivo o desenvolvimento de uma escala para a medição da percepção. Logo, as assertivas decorrem da identificação a partir da literatura e não houve uma avaliação qualitativa delas, exceto o pré-teste. Esta limitação é relevante pois, no caso de uso das assertivas para o desenvolvimento de estudos futuros, é preciso que os pesquisadores considerem esta limitação.

Cabe ainda destacar como limitações que o porte das empresas e os seus setores não foram investigados. Estas variáveis poderiam influenciar o uso das ferramentas, o que direciona para mais uma oportunidade de estudo futuro.

Surgem aqui novas oportunidades de estudos futuros para o aprimoramento desta escala e a sua efetiva validação. Acreditamos que por se tratar de um tema novo, sobre o qual ainda carecem estudos, este artigo apresenta resultados preliminares que podem motivar o desenvolvimento desta área de conhecimento, sobre a qual novos estudos se mostram necessários. Destacamos aqui mais uma vez que modificações nos padrões de relacionamento pessoal e profissional impostos à sociedade global no ano de 2020 em decorrência do Covid-19 tornam ainda mais relevantes os estudos futuros sobre o uso de ferramentas tecnológicas de comunicação e seus impactos no ambiente organizacional.

\section{REFERÊNCIAS}

ALMEIDA, M. E. B. Letramento digital e hipertexto: contribuições à educação. In: PELLANDA, N. M. C.; SCHLÜNZEN, E. T. M.; SCHLÜNZEN JUNIOR, K. (org.). Inclusão digital: tecendo redes afetivas / cognitivas. Rio de Janeiro: DP\&A, 2005. cap. 11.

ALTER, S. Work system theory: overview of core concepts, extensions, and challenges for the future. Journal of the Association for Information Systems, v. 14, n. 2, 72-121, fev. 2013.

AMATO NETO, J. Redes de cooperação produtiva e clusters regionais: oportunidade para as pequenas e médias empresas. São Paulo: Atlas, 2000.

ARAUJO, D. C., Simanski, E. S. S., \& QUEVEDO, D. M. Comunicação interna: relação entre empresa e colaboradores, um estudo de caso. BBR - Brazilian Business Review, v. 9, n. 1, 47-64, jan./mar. 2012. 
BH PRESS. Comunicação interna 3.0 e as mudanças de paradigmas. Disponível em http://www.presscomunicacao.com.br/comunicacao-interna/comunicacao-interna-3-0/2018. Acesso em: 30 abr. 2020.

BRANDÃO, J. M. F., TEMOTEO, J. A. G., \& CÂNDIDO, G. A. A união faz a força: análise do processo de aprendizagem interorganizacional em uma rede paraibana de hotéis. Revista Brasileira de Pesquisa em Turismo, v. 12, n. 2, p. 25-4, 2018.

BUENO, B., \& BALESTRIN, A. Inovação colaborativa: uma abordagem aberta no desenvolvimento de novos produtos. RAE - Revista de Administração de Empresas, v. 52, n. 5, p. 517-530, 2012.

CAETANO, J. M. M. Estilo de liderança e relações interpessoais e intergrupais em contexto escolar (Dissertação de Mestrado, Universidade Aberta), 2005. Disponível em https://repositorioaberto.uab.pt/bitstream/10400.2/672/1/LC183.pdf. Acesso em: 30 abr. 2020.

CALIFANO, B. Políticas de comunicação: desde as origens até os desafios das tecnologias digitais. Revista Mexicana de Opinião Pública, v. 25, 133-150, dez. 2018.

CARDOSO, T. P. et al. A aplicação do e-commerce no ramo agropecuário: uma estratégia tecnológica para a inovação organizacional. [2017]. Disponível em:

http://login.semead.com.br/20semead/anais/resumo.php?cod_trabalho=1743. Acesso em: 30 abr. 2020.

CARVALHO, H.; DUARTE, S.; MACHADO, V. C. Lean, agile, resilient and green: divergencies and synergies. International Journal of Lean Six Sigma, v. 2, n. 2, p. 151-179, 2011.

CETIC. Centro Regional de Estudos para o Desenvolvimento da Sociedade da Informação. TIC Empresas 2017. [2017]. Disponível em https://cetic.br/tics/empresas/2017/empresas/B18/. Acesso em: 30 abr. 2020.

COSTA, E. S. Com quantos gigabytes se faz uma jangada, um barco que veleje: o Ministério da Cultura, na gestão Gilberto Gil, diante do cenário das redes e tecnologias digitais. (Dissertação de Mestrado, Fundação Getúlio Vargas), 2011. Disponível em https://bibliotecadigital.fgv.br/dspace/handle/10438/8437?show=full

DANCEY, C. P., \& REIDY, J. Estatística sem matemática para psicologia. Porto Alegre: Bookman, 2006.

DUARTE, E. A.; BRAGA, R. M. O. A comunicação organizacional em unidades de informação. Informação \& Informação, v. 17, n. 3, 2012.

FONTANELLA, P. Comunicação e inovação: reflexões contemporâneas. RAC - Revista de Administração Contemporânea, v. 14, n.6, 1.171-1.173, dez., 2010.

FONTANINI, J. I. C.; CARVALHO H. G. As inovações incrementais em processos e seus fatores contribuintes em um ambiente industrial: um estudo de caso. In: ENCONTRO NACIONAL DE ENGENHARIA DE PRODUÇÃO, 25. 2005, Porto Alegre. Anais do Encontro Nacional de Engenharia de Produção. Porto Alegre: PUC, 2005.

HAIR Jr., J. F. et al. Análise multivariada de dados. 6. ed) Porto Alegre: Bookman, 2009.

KORGAONKAR, P. K.; WOLIN, L. D. A multivariate analysis of web usage. Journal of Advertising Research, v. 39, n. 2, p. 53-68, mar./abr. 1999.

KUNSCH, M. M. K. Gestão estratégica em comunicação organizacional e relações públicas. São Paulo: Difusão, 2008.

LACOMBE, F. J. M. Recursos humanos: princípios e tendências. 2. ed. São Paulo: Saraiva, 2012.

LARA, I. C. Jogando com a matemática de 5ª a 8ª série. São Paulo: Rêspel, 2009. 
LEITÃO, S. P., FORTUNATO, G., \& FREITAS, A. S. Relacionamentos interpessoais e emoções nas organizações: uma visão biológica. Revista de Administração Pública, v. 40, n. 5, p. 883-907, out. 2006.

MAILHIOT, G. B. Dinâmica e gênese dos grupos: atualidade das descobertas de Kurt Lewin. 1. ed. São Paulo: Vozes, 2013.

MARTINO, L. M. S.; MARQUES, C. S. Teorias da comunicação: processos, desafios e limites. São Paulo: Plêiade, 2015.

MASCARENHAS, A. O.; VASCONCELOS, F. C. Tecnologia na gestão de pessoas: estratégias de autoatendimento para o novo RH. São Paulo: Pioneira Thomson Learning, 2004.

MASCARENHAS, S. A. Metodologia científica. São Paulo: Pearson Education do Brasil, 2012.

MATOS, G. G. Comunicação sem complicação: como simplificar a prática da comunicação nas empresas. Rio de Janeiro: Elsevier, 2004.

MONTEIRO, C., KARPINSKI, J. A., \& ANGNES, J. S. A comunicação organizacional interna: um estudo no Programa Nacional de Formação em Administração Pública da Universidade Estadual do Centro Oeste. Revista de Administração IMED, v.5, n.2, 121-138, 2015.

OCDE. ORGANIZAÇÃO PARA COOPERAÇÃO E DESENVOLVIMENTO ECONÔMICO. Manual de Oslo: diretrizes para a coleta e interpretação de dados sobre inovação tecnológica. São Paulo: FINEP, 2005.

TASSIGNY, M. M., BRASIL, M. V. O., BUGARIN, M. C., \& NOGUEIRA, F. L. B. M. Blog: ferramenta estratégica de comunicação organizacional. Revista de Administração UFSM, v. 5, n. 2, p. 262-276, maio/ago. 2012.

TIDD, J.; BESSANT, J. Gestão da inovação. Porto Alegre: Bookman, 2015.

ZEFFANE, R.; TIPU, S. A. A.; RYAN, J. C. Communication, commitment \& trust: exploring the triad. International Journal of Business and Management, v. 6, n. 6, p. 77-87, 2011. 